\title{
Current Knowledge on Tumour Markers in Veterinary Oncology
}

\section{Gamze Bilgili ${ }^{1}$, Merve Alpay ${ }^{2}$, Deniz Ceylanli ${ }^{3}$, Sevgi Gençosman ${ }^{3}$, Çağri Gültekin ${ }^{4}$, Ahmet Özer Şehirli ${ }^{5}$ and Serkan Sayiner ${ }^{3 *}$}

${ }^{1}$ Faculty of Veterinary Medicine, Near East University, 99138 Nicosia, North Cyprus, Mersin 10, Turkey

${ }^{2}$ Department of Biochemistry, Faculty of Medicine, Duzce University, 81620 Düzce, Turkey

${ }^{3}$ Department of Biochemistry, Faculty of Veterinary Medicine, Near East University, 99138 Nicosia, North Cyprus, Mersin 10, Turkey

${ }^{4}$ Department of Surgery, Faculty of Veterinary Medicine, Near East University, 99138

Nicosia, North Cyprus, Mersin 10, Turkey

${ }^{5}$ Department of Pharmacology, Faculty of Dentistry, Near East University, 99138

Nicosia, North Cyprus, Mersin 10, Turkey

*Corresponding Author: Serkan Sayiner, Associate Professor, Department of Biochemistry, Faculty of Veterinary Medicine, Near East University, 99138 Nicosia, North Cyprus, Mersin 10, Turkey.
Received: December 20, 2021

Published: January 31, 2022

(C) All rights are reserved by Serkan Sayiner., et al.

\begin{abstract}
Tumour markers, also known as biomarkers, might be proteins, conjugated proteins, peptides, or carbohydrates. Tumour markers are substances that are created by cancer cells or by the organism in response to cancer. According to the dictionary of cancer terms published online by the National Cancer Institute (NCI), a biomarker is "A biological molecule found in blood, other body fluids, or tissues that is a sign of a normal or abnormal process, or of a condition or disease." These molecules are present in the blood, urine, tissues, and body fluids (cerebrospinal fluid, synovial fluid, pancreatic fluid, etc.). However, blood levels are primarily examined. Tumour markers are never utilized to make a cancer diagnosis. Mammography, ultrasound, computed tomography, magnetic resonance imaging scans, and tumour marker assays are used to aid in the diagnosis, monitor the progression of the disease (prognosis), detect recurrence, and aid in the application of treatment. While these procedures are beneficial for staging cancer, a biopsy is usually required to confirm the diagnosis. Considering tumour markers is an exciting field for both veterinary oncology and human medicine, the purpose of this study is to provide current and relevant information for the future use of tumour markers for the diagnosis and prognosis of increasing cancer cases in animals by reviewing previous studies.
\end{abstract}

Keywords: Cancer Antigen; Oncofetal Antigens; PSA; Tumour markers; Veterinary Oncology

\section{Introduction}

While neoplasms, which are defined as abnormal, rapid, and uncoordinated new tissue growths, can be benign or malignant, cancer is defined as malignant growths. Neoplastic cells vary from normal cells by their independent and unregulated growth, which results in defective cellular differentiation, abnormal cell communication, and adhesion. The causes of the slow accumulation of errors in cellular DNAs can be explicitly identified as oncogenes or tumour suppressor genes. External factors including viruses, parasites, hormones, chemical agents, ultraviolet light, and trau- ma/chronic inflammations are common causes of the presence of genetic alterations in animals [30].

Malignant neoplasms can spread locally, invasively, and destructively to other areas of the body. Even though some canine tumours are malignant, metastasis may be uncommon. Evaluation of histological type and grading or appearance (size) of tumours that can metastasize to lymph nodes or hematogenous pathways is important in prognosis. At the initial examination, the extent or stage of the disease, as well as the investigation of complications and any concurrent conditions, should be conducted. A definitive 
diagnosis is acquired in histological evaluation through microscopic inspection of biopsy tissue. Today, comparative approaches to understanding cancer and, as a result, better diagnosing, preventing, and treating cancer is common and effective. Tumour markers are proteins that are induced by the activity of organism cells or cancer cells, and they have become a useful test, particularly in human medicine, with the evaluation of their activities in diagnostic procedures, differential diagnosis, prognosis, and post-treatment [37]. The presence of promising tumour indicators in veterinary medicine is exciting. This study is intended to review tumour indicators that can be used in the field of veterinary oncology based on prior research.

\section{Tumour markers}

Tumour markers, which are detected in cells, tissues, and body fluids as a specific response to tumours or cancer cells, are an interesting tool for the clinical discipline of oncology as they can be quantified using chemical, immunological, molecular, and mass spectrometry techniques. Although the use of tumour markers for screening is not feasible due to the high sensitivity and specificity required, they are useful for diagnosing cancer and detecting recurrence following treatment, however, they are insufficient for diagnosis. Because measured levels may be indicative of malignant forms or maybe produced for benign reasons, it is necessary to conduct a careful analysis of specific and nonspecific increases [36]. Today, various tumour markers are a research subject in veterinary oncology (Table 1).

Although the characteristics of an ideal tumour marker vary according to classification and application, general characteristics include the ability of the marker to be produced at specific and detectable levels by premalignant or malignant tissue, allowing for diagnosis even in the early stages of the disease, and it should be organ-specific and non-invasive. It should demonstrate its existence in tissue, quantify levels associated with tumour volume, biological behaviour, or disease development, reflect temporal changes in tumour burden and response to treatment, and be standardized, reproducible, and demonstrated to be accurate. On the other hand, it should be safe, inexpensive, high yielding, with a low false-positive rate (FPR) and reasonably low false-negative rate (FNR) [29].

\section{Role of tumour markers in screening}

Due to their low sensitivity and lack of specificity, tumour markers have limited relevance in tumour screening. The level is not elevated sufficiently in the early stages of the disease for the marker to identify it. In this instance, the marker is unable to aid in diag- nosis. Additionally, numerous tumour markers are raised during benign/inflammatory illnesses of the relevant organ. As a result, it produces false-positive results. When a test's sensitivity is great, false-negative findings are minimized. When a test's specificity is high, false-positive findings are minimized [30].

\section{Role of tumour markers in the diagnosis}

Although tumour markers are not very important diagnostic tools, they should be used in conjunction with other research and a complementary manner. Most tumour marker values are not sufficient to diagnose cancer. In some benign conditions, the amount of the marker may increase. Levels may not be elevated in every cancer patient. In addition, other types of cancer may play a role in increasing the level of a tumour marker [36].

Role of tumour markers in monitoring prognosis and recurrence

One of the most useful applications of tumour markers is their usefulness in prognosis and recurrence monitoring. The level of serum tumour markers is a good indicator of the treatment's efficacy. A high marker level following surgical excision of tumour tissue indicates the likelihood of partial removal, recurrence, or metastasis. If the tumour marker does not reduce or rise following surgery, it is assumed that the malignancy did not respond to treatment or that there is still tumour tissue present. While tumour markers are incredibly valuable for detecting tumour therapy response or recurrence, most tumour markers are not sensitive enough [29].

\begin{tabular}{|c|c|c|}
\hline Group & Marker & Diagnostic Value \\
\hline \multirow[t]{2}{*}{ Oncofoetal antigens } & $\begin{array}{c}\text { Alpha-fetoprotein } \\
\text { (AFP) }\end{array}$ & $\begin{array}{l}\text { Hepatocellular } \\
\text { carcinoma }\end{array}$ \\
\hline & $\begin{array}{c}\text { Carcinoembryonic } \\
\text { antigen (CEA) }\end{array}$ & $\begin{array}{c}\text { Undefined in } \\
\text { veterinary } \\
\text { oncology }\end{array}$ \\
\hline \multirow[t]{3}{*}{$\begin{array}{l}\text { Tumour-Associated } \\
\text { Antigens }\end{array}$} & $\begin{array}{l}\text { Cancer antigen } \\
\text { (CA) 19-9 }\end{array}$ & $\begin{array}{c}\text { Pancreatic } \\
\text { carcinoma, } \\
\text { cholangiocarcinoma, } \\
\text { not } \\
\text { applicable in } \\
\text { veterinary } \\
\text { medicine }\end{array}$ \\
\hline & CA-125 & $\begin{array}{c}\text { not applicable in } \\
\text { veterinary } \\
\text { medicine }\end{array}$ \\
\hline & CA15-3 & $\begin{array}{c}\text { Spontaneous } \\
\text { mammary gland } \\
\text { tumours }\end{array}$ \\
\hline
\end{tabular}




\begin{tabular}{|c|c|c|c|c|}
\hline & CA-50 & $\begin{array}{c}\text { not applicable in } \\
\text { veterinary medicine }\end{array}$ & \multirow[t]{2}{*}{ Fibronectin } & \multirow{2}{*}{$\begin{array}{c}\text { No functional } \\
\text { distinction of FN } \\
\text { concentrations }\end{array}$} \\
\hline & & Canine prostatic & & \\
\hline \multirow{5}{*}{ Enzymes } & \multirow[b]{2}{*}{$\begin{array}{c}\text { Prostatic acid } \\
\text { phosphatase } \\
\text { (PAP) }\end{array}$} & \multirow{2}{*}{$\begin{array}{l}\text { Increase of PAP does } \\
\text { not appear to occur } \\
\text { in dogs with prostate } \\
\text { cancer }\end{array}$} & Alpha (TNF- $\alpha$ ) & \\
\hline & & & \multirow[t]{2}{*}{$\begin{array}{c}\text { Cyclooxygenase- } 2 \\
\text { (Cox-2) }\end{array}$} & \multirow{2}{*}{$\begin{array}{l}\text { Feline and canine } \\
\text { invasive } \\
\text { micropapillary } \\
\text { carcinomas (IMCs) }\end{array}$} \\
\hline & $\begin{array}{c}\text { Alkaline } \\
\text { phosphatase } \\
\text { (ALP) }\end{array}$ & $\begin{array}{l}\text { Appendicular } \\
\text { osteosarcoma }\end{array}$ & & \\
\hline & $\begin{array}{l}\text { Neuron-specific } \\
\text { enolase (NSE) }\end{array}$ & $\begin{array}{l}\text { Neuroblastoma, } \\
\text { small cell lung } \\
\text { cancer }\end{array}$ & Vascular endothe- & Hepatocellular \\
\hline & $\begin{array}{c}\text { Thymidine kinase } \\
\text { (TK) }\end{array}$ & Canine lymphoma & $\begin{array}{c}\text { lial growth factor } \\
\text { (VEGF) }\end{array}$ & $\begin{array}{c}\text { carcinoma (HCC) } \\
\text { metastasis }\end{array}$ \\
\hline
\end{tabular}

Table 1: Tumour markers in veterinary oncology practice.

\section{Oncofoetal antigens}

Oncofoetal proteins are produced by tumour cells. It enters the bloodstream as a degradation product, either secreted by the tumour or released as a by-product of tumour cell breakdown. During embryogenesis, oncofoetal proteins are already present, but are detectable in adults in disease states. The most often utilized oncofoetal protein tumour indicators are CEA and AFP [40].

Serum AFP levels are significantly greater in canine hepatocellular carcinoma than in other hepatic disorders in dogs. Therefore, it can be an extremely useful tool for identifying canine hepatocellular cancer. On other domesticated animal species, serum AFP has not been thoroughly evaluated as a tumour marker. AFP has also been detected in the serum of cancer-stricken dogs. However, the mean serum AFP concentrations in dogs with various malignancies did not differ significantly from those in healthy and cancer-free dogs. AFP may contribute to the absence of other blood biochemical abnormalities and the diagnosis of primary or secondary liver cancer [23].

CEA has also been extensively explored in human medicine, where it has been used as a significant marker for lung, colon-rec- 
tum, breast, ovarian, and prostate gland cancers. CEA has received attention last years in veterinary oncology. Mammary tumours are one of the most common fatal tumours in female dogs. In veterinary medicine, dogs with mammary gland tumours have higher CEA levels compared to healthy dogs but have low specificity. The reason for this may increase due to colorectal cancer, lung cancer and pancreatic cancers as in humans [9].

\section{Tumour-associated antigens}

Tumour-associated antigens are seen in a distinct type of tumours within the same tissue type, which is mostly produced in mice and less commonly in rabbits, differ in terms of specificity, affinity, antigen absorption, and the necessity for additional treatments [37].

CA15-3 is a mucin glycoprotein with a large molecular weight that is encoded by the Mucin 1(MUC1) gene and is involved in cell adhesion. Although the most common causes of CA15-3 increase are Cobalamin (vitamin B12) deficiency and renal failure, it is a widely used serum marker in mammary tumour cancer. The determination and monitoring of serum CEA, CA15-3, and LDH concentrations in female dogs with spontaneous mammary gland tumours is relevant for evaluating the disease's stages [9]. Although the most common causes of CA15-3 increase are vitamin $B_{12}$ deficiency and renal failure, it is a widely used serum marker in breast cancer. CA15-3 is a mucin glycoprotein with a large molecular weight that is encoded by the MUC1 gene and is involved in cell adhesion. The determination and monitoring of serum CEA, CA15-3, and LDH concentrations in female dogs with spontaneous mammary gland tumours is relevant for evaluating the disease's stages [6].

PSA, which has become a frequently used tumour marker in prostate cancer screening programs, is a tumour-associated antigen. It is detected in the serum of males with prostate cancer, as well as prostate hyperplasia, which is common in intact male dogs during ageing. This indicates that benign prostate gland enlargement may be indistinguishable from malignancy. So, it is controversial to use as diagnostic marker for prostatic tumour of dog [11].

The p53 protein is necessary for cell proliferation regulation. Typically, aberrant deposition is observed in cutaneous cancers. Immunolabeling was used to investigate p53 in canine cutaneous endothelium tumours. The size of the tumour has been found to correlate with the hemangiosarcoma stage (dermal and hypoder- mal), histological diagnosis, and proliferative activity. However, hemangiosarcoma's demonstrated a considerable increase in p53 immunoactivity against haemangiomas. The p53 index was related to a significantly shorter survival time in the Kaplan-Meier survival analysis. p53 gene mutations and protein overexpression using the Polyclonal antibody (pAb) anti-p53 antibody, among other findings, are regarded to be useful indicators of greater malignant potential and poor prognosis in canine mammary cancers. p53, ER, and Ki67 were studied in 170 dogs with breast cancer, and it was discovered that the three markers can be employed in addition to histological grade to control the biological behaviour of canine mammary carcinomas [4].

\section{Enzymes}

Enzyme markers are assays that are used to evaluate the activity of certain enzymes in the bodies of live organisms. Diseases, abnormalities, or anomalies of genetic origin (inherited) can impact the way enzyme's function. Lactate Dehydrogenase (LDH), Serum Alkaline Phosphatase (ALP), PAP, and Thymidine kinase (TK) are all members of this category.

LDH activity is elevated in dogs with lymphoma that lacks isoenzyme activity; it is an important marker for canine lymphoma since dogs with lower serum LDH activity often have longer life periods than dogs with increased LDH activity [26].

ALP, that has liver, bone, and adrenocortical isoenzymes, is thought to be a predictive factor in animals with various cancers, such as osteosarcoma, soft tissue sarcoma and mammary gland tumours. Total ALP activity especially increases in mammary gland tumours. However, isoenzyme analysis may help to predict the type of tumour [21].

PAP was commonly utilized as a tumour diagnostic. Serum PAP activity is elevated in individuals with prostate cancer and plays a critical role in prostate carcinogenesis. PAP activity is not increased in dogs with prostate cancer, so it is controversial [1].

TK is an enzyme that acts as a phosphotransferase in most live cells. TK is a tumour marker because it exhibits lower serum activity following surgical resection of the main tumour in women with breast cancer. Additionally, it can be used to determine prognosis in patients with non-Hodgkin lymphoma and multiple myeloma. Serum TK activity appears to be a predictor of survival in dogs with 
lymphoma. Because TK may be determined using non-radiometric methods, it is more suitable for routine laboratory use [8].

\section{Hormones}

Hormone levels may explain differences in risk for several of the most frequently diagnosed malignancies, including breast, ovarian, and other cancers, according to recent research. Nonetheless, additional studies and programs utilizing novel hormone measurement technologies are required to deduce and comprehend the roles of common hormones found in many types of cancer. The function of hormones and other biomarkers in cancer aetiology has enormous potential to aid in our understanding of various diseases, facilitate the development of new treatment methods, and maximize the benefit of detection [22].

Inhibin is a nonsteroidal protein hormone that is released by male Sertoli cells and female granulosa cells. Inhibin works by suppressing the synthesis and release of follicle-stimulating hormone (FSH) in the pituitary gland and decreasing the LH-releasing hormone level in the hypothalamus. Inhibin regulates the follicular phase of the horse's estrus cycle [27]. Mares with granulosa theca cell tumours (GTCT) have significantly higher serum inhibin levels [35]. Increased blood inhibin concentrations in dogs with Sertoli cell tumours have the potential to aid in the identification and diagnosis of testicular tumours. Besides, elevated levels of inhibin can be detected in adrenocortical tumours and pituitary-dependent hyperadrenocortisism [3].

When hypercalcemia is present, increased serum parathyroid hormone (PTH) is considered adequate to diagnose primary hyperparathyroidism usually. However, retrospective case-control research reveals that mean PTH levels will be an abnormal physiological response of the parathyroid gland in hypercalcemia, consistent with primary hyperparathyroidism [10]. While primary hyperparathyroidism in dogs and cats is uncommon, both adenomas and carcinomas of the parathyroid glands have been recorded in the literature. Additionally, it should be considered as a differential diagnosis.

Malignant hypercalcemia is a well-known paraneoplastic condition that affects animals, specifically dogs and cats. This is the most prevalent cause of dog hypercalcemia that persists. The synthesis of PTH-associated protein elucidates a large portion of the humoral hypercalcemia associated with malignancy (PTHrP). Increased se- rum PTHrP concentrations have been identified in a dogs and cats with malignant humoral hypercalcemia associated with different carcinomas and lymphomas [2].

The diagnosis of hyper-functional adrenocortical tumours containing this hormone can be made by stimulating natural or synthetic adrenocorticotropin hormone (ACTH) or by surveying the basal plasma cortisol concentration or the plasma cortisol concentration following various provocative tests using dexamethasone suppression. Endogenous ACTH measures may assist in differentiating basic adrenal irregularities from secondary ones caused by pituitary gland malfunction. Precursor hormones include pro-opiomelanocortin and pro-adrenocorticotropin. In dogs with hyperadrenocorticism, the ratio of these two hormones has been related with the size of pituitary tumours [12]. Provocative ACTH testing and basal ACTH monitoring have been used to diagnose pars intermedia adenomas in the horse's pituitary [24].

Plasma sex steroid hormones are analyzed to assist describe GTCT in mares exhibiting clinical symptoms. Significant discrepancies exist in the sex steroid hormone profiles of horses with elevated plasma progestin levels. This difference could be due to aberrant ovarian follicles, aromatase activity abnormalities, or potential feedback inhibition impairing gonadotropin secretion [19]. Understanding the plasma concentrations of androstenedione and 17-hydroxyprogesterone is significant. Additionally, oestradiol may aid in the diagnosis of ferrets suffering from adrenocortical neoplasia. Provocative testing with ACTH has also been shown to raise plasma concentrations of these adrenal sex hormones in dogs with adrenal gland cancer [16].

Progesterone levels were elevated in the plasma of a cat with well-differentiated adrenocortical cancer. There is a substantial body of literature on oestrogen and progesterone receptors in canine mammary gland tumours. Recent findings and studies reveal that androgen serum and tissue concentrations rise in dogs with physiologically aggressive inflammatory breast cancer vs dogs with non-inflammatory malignant breast tumours [20]. Progesterone receptors have been identified in equine lymphomas. A case report of cutaneous lymphoma regression in a mare following GTCT excision demonstrates the biological significance of progesterone receptors in horse lymphomas [15].

Insulin is a hormone having, a polypeptide structure, and a role in carbohydrate degradation regulation in the body in conjunction 
with glucagon. This hormone has a hypoglycaemic impact. Insulinoma (beta cell tumour, pancreatic islet cell tumour) is defined by increased insulin release from the pancreas beta cells. Although the insulin-glucose rate varies widely in the literature, the aberrant physiological state of fasting hypoglycemia and a detectable serum insulin ratio is considered adequate to diagnose insulinoma. Serum fructosamine and glucose haemoglobin levels can also aid in the diagnosis of insulinoma. Lower blood fructosamine levels and decreased serum glycoside haemoglobin levels was detected in numerous small dogs with insulinoma. Even in the presence of normoglycemia, a low serum fructosamine level may suggest the presence of insulinoma [28].

Thyroid hormones include thyroxine, triiodothyronine, and calcitonin, which are generated and secreted by the thyroid gland. These three hormones are produced by tyrosine and are involved in metabolic regulation. Triiodothyronine $\left(\mathrm{T}_{3}\right)$ and Thyroxine $\left(\mathrm{T}_{4}\right)$ are primarily made up of iodine. $\mathrm{T}_{4}$ regulates the metabolic rate and is intimately connected with digestion, heart and muscle function, brain development, and bone health. Serum thyroglobulin levels were elevated in $70 \%$ of dogs with thyroid cancer, but there was no substantial association with serum $\mathrm{T}_{4}$ levels. Although elevated blood total or free $\mathrm{T}_{4}$ levels are frequently used to diagnose hyperthyroidism in cats, elevated free $\mathrm{T}_{4}$ levels can be detected in certain cats with disorders other than thyroid [33]. Additional testing with the $\mathrm{T}_{3}$ suppression test may be necessary to establish the diagnosis of hyperthyroidism in cats with doubtful results [13]. In many cats with hyperthyroidism, a functional adenoma or adenomatous hyperplasia should be considered before a basic thyroid cancer is considered. Serial $\mathrm{T}_{4}$ measurements are useful for evaluating treatment response and diagnosing relapse following radioiodine therapy [32].

\section{Immunoglobulins}

Serum immunoglobulins have been measured in veterinary medicine for a long period. Hyperglobulinemia is a symptom of B cell disorders, such as multiple myeloma and Waldenström's macroglobulinemia. Electrophoresis of serum proteins results in the creation of monoclonal gammopathy, and immuno-electrophoresis is used to assess the antibody class of immunoglobulins. Because hyperglobulinemia frequently improves following successful therapy, the response to serum immunoglobulin measurement is appropriate for determining recurrence. In dogs, Immunoglobulin $\mathrm{M}$ (IgM), Immunoglobulin G (IgG), and Immunoglobulin A (IgA) gam- mopathies were detected While hypergammaglobulinemia does not always occur in myeloma, hypogammaglobulinemia can occur with a consistent electrophoretic pattern. Infection with Ehrlichia can also result in a monoclonal gammopathy in dogs, and increased serum immunoglobulins should be considered a differential diagnosis [25].

Various serum tumour markers

Acute phase proteins (APPs)

APP are proteins whose concentrations fluctuate in response to tissue injury or infection. APP is mostly composed of plasma proteins synthesized by hepatocytes. They have a lengthy history of being associated with cancer in human medicine. Increased APP levels at diagnosis are a poor prognostic sign in cancer patients. Increased serum C-Reactive Protein (CRP) levels, for example, are a poor prognostic sign in patients with kidney, pancreatic, gastrointestinal system, lymphoma, multiple myeloma, melanomas, and ovarian malignancies. Interleukin 6 (IL-6) and tumour necrosis factor-alpha (TNF- $\alpha$ ) both promote the synthesis of CRP and maybe other APPs, making APPs attractive as biomarkers and therapeutic targets. Additionally, APP has been investigated in veterinary medicine. Significant increases in Ceruloplasmin (Cp), Haptoglobin (Hp), and CRP levels were reported in dogs with cancer and other inflammatory disorders in a recent APP study, and their reduction after therapy was related to improved outcomes [39]. Serum concentrations of Serum Acid Alpha-1-glycoprotein (AGP) were found to decrease with the initiation of cancer treatment and clinical remission in dogs with lymphoma, implying that APP may serve as a biomarker and may be therapeutic [14].

\section{Fibronectin (FN)}

FN is an extracellular matrix component as well as a major glycoprotein present in plasma in its soluble form. Fibronectin molecules aggregate only on the surfaces of cells and require the addition of additional proteins to create filaments. Fibronectin is found in blood and other body fluids in various isoforms. It is known to aid in blood clotting, wound healing, and phagocytosis. Plasma FN concentrations in dogs with malignancies were shown to be both elevated and lowered. No functional distinction of FN concentrations between malignant and non-malignant pleural and abdominal effusions was observed in a study of canine and feline pleural and abdominal effusions. However, dogs with malignant effusion had a higher FN/albumin ratio than dogs with congestive heart failure [17]. 
Sialic acid

Sialic acid identifies neuraminic acid derivatives that may be useful as tumour markers in cancer cells with aberrant glycosylation. Sialic acid has been shown to have a high cancer specificity, but a low sensibility due to the presence of sialic acid-rich glycoproteins in inflammatory disorders. Sialic acid is a possible diagnostic marker in dogs suffering from mammary tumours [31].

\section{Tumour necrosis factor alpha (TNF- $\alpha$ )}

While TNF's primary purpose is to regulate immune cells, it is also an endogenous pyrogen that can induce fever, apoptosis, cachexia, and inflammation, inhibit carcinogenesis and viral replication, and respond to sepsis via cells that produce IL-1 and IL6. In addition to playing a role as a cell signal protein in systemic inflammation, it is also one of the cytokines responsible for initiating the acute phase response. TNF has a 3-monomer glycoprotein structure consisting of 212 amino acids synthesized by monocytes and macrophages. Its membranous and soluble forms are physiologically active. Following the warning, the signal travels through three major paths. The first two interact with nuclear factor-kappa (NF-k) and activator protein-1 specifically during inflammation, and in a third method, during apoptotic formation. Because of its pleiotropic effects, it is responsible for different effects on different cells at different stages throughout inflammatory disorders. Even though human and canine TNF- $\alpha$ share $91 \%$ similarity, the EnzymLinked ImmunoSorbent Assay (ELISA) test failed to detect canine TNF- $\alpha$. Additionally, it appears that the serum has limited utility as a tumour marker in dogs with TNF- $\alpha$ lymphoma [18].

\section{Cyclooxygenase-2 (COX-2)}

COX-2, the enzyme responsible for the conversion of arachidonic acid to prostaglandins, is overexpressed in a variety of tumour types, including colon, pancreatic, lung, head, and neck malignancies. COX-2 is an inducible enzyme that has been linked to tumour growth and angiogenesis. COX-2 overexpression and tumour differentiation were associated with increased COX-2 expression in dogs, but still needs more study in veterinary oncology. Besides, it also elevates due to inflammation, fever and pain [38].

\section{Caveolin-1 (Cav-1)}

Caveolins are the caveolae's primary structural proteins and are involved in tumour surveillance. Increased Cav-1 expression has been linked to neoplasia. The Cav-1 expression has been linked to malignant canine mammary tumours, their vascular invasion, and regional lymph node metastases. Cav-1 was observed to be overexpressed in malignant and invasive CMT patients, and it was speculated that Cav-1 could be effective in predicting CMT malignancy $[7]$.

\section{Telomerase activity}

Telomeres are structures found at the ends of linear chromosomes. Sequential cell divisions occur as a result of Deoxyribonucleic Acid (DNA) polymerase's failure to synthesis the end of the linear DNA, and telomeres become increasingly shorter as a result. Telomerase adds new telomeric sequences to the end of chromosomal DNA to correct a mistake that occurred during the previous replication. Unlike normal somatic cells, many human tumours have telomerase activity. The findings of a study that called into question the value of telomerase activity as a marker for canine tumours revealed that telomerase could be a helpful diagnostic marker as well as a target for antitumour treatment. Following the demonstration of the diagnostic significance of telomerase activity in humans and dogs, it was shown that the TRAP test in cats could be effective in providing a quick diagnosis of cancer [5].

\section{Vascular endothelial growth factor (VEGF)}

VEGF, a regulator of angiogenesis and vascular permeability, can be evaluated as a prognostic marker and maybe a future therapeutic target. It was mentioned in a study examining VEGF expression in canine cerebral meningiomas that it was quantifiable and may be associated with bad results [34].

\section{Conclusion}

Numerous tumour markers can be increased in non-cancerous or disease-related circumstances. Certain tumour markers are unique to types of cancer, whereas others are seen in a variety of different types of cancer. Not every patient diagnosed with a particular type of cancer will have a high level of the tumour marker in question. Not every cancer has been linked to a tumour marker. Despite all this information regarding tumour markers, there is still no optimal tumour marker available today. Apart from the above-mentioned perfect tumour marker qualities, the use of these markers in diagnostics will be extremely convenient in both veterinary and human medicine. A timely and accurate diagnosis enables more effective therapy and, of course, a better prognosis. Thus, the patient's welfare is improved, and it is no longer essential to obtain 
a biopsy sample for diagnosis. Nowadays, tumour marker testing can provide beneficial information, but it has its limitations. Sufficient resources and funds should be directed to the development of tumour markers.

\section{Acknowledgements}

We would like to express our gratitude to Handan Gözde Süer for her support.

\section{Conflict of Interest}

All authors declare that they have no conflicts of interest.

\section{Bibliography}

1. Amorim RL., et al. "Serum and urinary measurements of prostatic acid phosphatase (PAP) and prostatic specific antigen (PSA) in dogs". Arquivo Brasileiro de Medicina Veterinária e Zootecnia 56.3 (2004): 320-324.

2. Bolliger AP., et al. "Detection of parathyroid hormone-related protein in cats with humoral hypercalcemia of malignancy". Veterinary Clinical Pathology 31.1 (2002): 3-8.

3. Brömel C., et al. "Serum Inhibin Concentration in Dogs with Adrenal Gland Disease and in Healthy Dogs". Journal of Veterinary Internal Medicine 27.1 (2013): 76-82.

4. Brunetti B., et al. "p53, ER, and Ki67 Expression in Canine Mammary Carcinomas and Correlation with Pathological Variables and Prognosis". Veterinary Pathology 58.2 (2021): 325331.

5. Cadile CD., et al. "Telomerase activity as a marker for malignancy in feline tissues". American Journal of Veterinary Research 62.10 (2001): 1578-1581.

6. Campos LC., et al. "CA15.3, CEA and LDH in Dogs with Malignant Mammary Tumors". Journal of Veterinary Internal Medicine 26.6 (2012): 1383-1388.

7. Ebisawa M., et al. "Significance of caveolin-1 and matrix metalloproteinase 14 gene expression in canine mammary tumours". The Veterinary Journal 206.2 (2015): 191-196.

8. von Euler HP., et al. "A non-radiometric method for measuring serum thymidine kinase activity in malignant lymphoma in dogs". Research in Veterinary Science 80.1 (2006): 17-24.

9. Fan Y., et al. "Combined detection of CA15-3, CEA, and SF in serum and tissue of canine mammary gland tumor patients". Scientific Reports 11.1 (2021): 6651.
10. Feldman EC., et al. "Pretreatment clinical and laboratory findings in dogs with primary hyperparathyroidism: 210 Cases (1987-2004)". Journal of the American Veterinary Medical Association 227.5 (2005): 756-761.

11. Golchin-Rad K., et al. "Changes in specific serum biomarkers during the induction of prostatic hyperplasia in dogs". BMC Veterinary Research 15.1 (2019): 440.

12. Granger N., et al. "Plasma Pro-opiomelanocortin, Pro-adrenocorticotropin Hormone, and Pituitary Adenoma Size in Dogs with Cushing's Disease". Journal of Veterinary Internal Medicine 19.1 (2005): 23-28.

13. Graves TK., et al. "Diagnostic tests for feline hyperthyroidism". Veterinary Clinics of North America - Small Animal Practice 24.3 (1994).

14. Hahn KA., et al. "Serum alpha 1-acid glycoprotein concentrations before and after relapse in dogs with lymphoma treated with doxorubicin... Journal of the American Veterinary Medical Association 214.7 (1999): 1023-5.

15. Henson KL., et al. "Regression of subcutaneous lymphoma following removal of an ovarian granulosatheca cell tumor in a horse". Journal of the American Veterinary Medical Association 212.9 (1998): 1419-1422.

16. Hill KE., et al. "Secretion of sex hormones in dogs with adrenal dysfunction". Journal of the American Veterinary Medical Association 226.4 (2005): 556-561.

17. Hirschberger J., et al. "Fibronectin concentrations in pleural and abdominal effusions in dogs and cats". Journal of Veterinary Internal Medicine 10.5 (1996): 321-325.

18. Hofer J., et al. "Detection of tumour necrosis factor-alpha in dogs with lymphoma*”. Veterinary and Comparative Oncology 9.4 (2011): 290-295.

19. Hoque S., et al. "Endocrinological changes before and after removal of the granulosa theca cell tumor (GTCT) affected ovary in 6 mares". Journal of Veterinary Medical Science 65.8 (2003): 887-891.

20. Illera JC., et al. "Steroids and receptors in canine mammary cancer". Steroids 71.7 (2006): 541-548.

21. Karayannopoulou M., et al. "Serum alkaline phosphatase isoenzyme activities in canine malignant mammary neoplasms with and without osseous transformation". Veterinary Clinical Pathology 35.3 (2006): 287-290. 
22. Key TJA. et al. "Hormones and cancer in humans". Mutation Research - Fundamental and Molecular Mechanisms of Mutagenesis 333.1-2 (1995): 59-67.

23. Kitao S., et al. "Alpha-Fetoprotein in Serum and Tumor Tissues in Dogs with Hepatocellular Carcinoma". Journal of Veterinary Diagnostic Investigation 18.3 (2006): 291-295.

24. van der Kolk JH., et al. "Laboratory diagnosis of equine pituitary pars intermedia adenoma.". Domestic animal endocrinology 12.1 (1995): 35-9.

25. Lautzenhiser SJ., et al. "Unusual IgM-secreting multiple myeloma in a dog". Journal of the American Veterinary Medical Association 223.5 (2003): 645-648+636.

26. Marconato L., et al. "Serum lactate dehydrogenase activity in canine malignancies". Veterinary and Comparative Oncology 7.4 (2009): 236-243.

27. Medan MS., et al. "Plasma concentrations of Ir-inhibin, Inhibin A, Inhibin pro- $\alpha \mathrm{C}, \mathrm{FSH}$, and estradiol- $17 \beta$ during estrous cycle in mares and their relationship with follicular growth". Endocrine 25.1 (2004): 7-14.

28. Morris J and Dobson J. "Small Animal Oncology".

29. Nagpal M., et al. "Tumor markers: A diagnostic tool". National Journal of Maxillofacial Surgery 7.1 (2016): 17.

30. Newkirk KM., et al. "Neoplasia and Tumor Biology". In: Pathologic Basis of Veterinary Disease Expert Consult. 2017:286321.e1.

31. Paltrinieri S., et al. "Sialic Acid and Sialyltransferase Activity in Serum and Tissues of Dogs With Mammary Tumors". Veterinary Pathology 49.4 (2012): 669-681.

32. Peterson ME., et al. "Radioiodine treatment of 524 cats with hyperthyroidism". Journal of the American Veterinary Medical Association 207.11 (1995): 1422-1428.

33. Peterson ME., et al. "Measurement of serum concentrations of free thyroxine, total thyroxine, and total triiodothyronine in cats with hyperthyroidism and cats with nonthyroidal disease". Journal of the American Veterinary Medical Association 218.4 (2001): 529-536.

34. Platt SR., et al. "Vascular endothelial growth factor expression in canine intracranial meningiomas and association with patient survival". Journal of Veterinary Internal Medicine 20.3 (2006): 663-668.
35. Renaudin CD., et al. "Equine granulosa cell tumours among other ovarian conditions: Diagnostic challenges". Equine Veterinary Journal 53.1 (2021): 60-70.

36. Sharma S., et al. "Tumor markers in clinical practice: General principles and guidelines". Indian Journal of Medical and Paediatric Oncology 30.01 (2009): 1-8.

37. Sokoll LJ., et al. "Tumor markers". In: Contemporary Practice in Clinical Chemistry. Elsevier; 2020:779-793.

38. Szweda M., et al. "Cyclooxygenase-2 as a biomarker with diagnostic, therapeutic, prognostic, and predictive relevance in small animal oncology". Journal of Veterinary Research 64.1 (2020): 151-160.

39. Tecles F., et al. "Preliminary Studies of Serum Acute-Phase Protein Concentrations in Hematologic and Neoplastic Diseases of the Dog". Journal of Veterinary Internal Medicine 19.6 (2005): 865-870.

40. Zhou L., et al. "Serum tumor markers for detection of hepatocellular carcinoma". World Journal of Gastroenterology 12.8 (2006): 1175-1181.

\section{Assets from publication with us}

- Prompt Acknowledgement after receiving the article

- Thorough Double blinded peer review

- Rapid Publication

- Issue of Publication Certificate

- High visibility of your Published work

Website: www.actascientific.com/

Submit Article: www.actascientific.com/submission.php

Email us: editor@actascientific.com

Contact us: +919182824667 